\title{
Terahertz Wave Transmission Properties of Metallic Periodic Structures Printed on a Photo-paper
}

\author{
Sung-Ho Lee and Sangyoon Gee \\ Graduate Program of Photonics and Applied Physics, GIST, Gwangju 500-712, Korea
}

Chul Kang and Chul-Sik Kee*

Nanophotonics Laboratory, Advanced Photonics Research Institute, GIST, Gwangju 500-712, Korea

(Received June 4, 2010 : revised July 29, 2010 : accepted August 16, 2010)

\begin{abstract}
We printed a one-dimensional array of metallic wires and a two-dimensional array of metallic split ring resonators on a photo-paper by using a high-dots-per-inch resolution printer and an ink with silver nano-particles. The printed sample sizes are $1.0 \times 1.0 \mathrm{~cm}^{2}$. The transmission measured by a terahertz time domain spectroscopy system shows that the arrays of wires and split ring resonators could act as polarizers and band-stop filters, respectively, in a terahertz frequency region.
\end{abstract}

Keywords: Terahertz waves, Photonic crystals, Wire grids, Split ring resonators

OCIS codes : (300.6495) Spectroscopy, terahertz; (160.5298) Photonic crystals

\section{INTRODUCTION}

Terahertz $(\mathrm{THz})$ waves are electromagnetic radiation with frequencies generally in a range of 0.1 to $10 \mathrm{THz}$, between the microwaves and the far-infrared [1]. Owing to recent advancements in optical and electrical components, efficient $\mathrm{THz}$ sources, detectors, and passive devices have been developed [2]. THz time-domain spectroscopy (THz-TDS) and $\mathrm{THz}$ imaging systems still demand suitable $\mathrm{THz}$ components for improving their performance.

Artificial periodic structures have been demonstrated to be useful in manipulating electromagnetic waves. The representative example is a photonic crystal which is periodically composed of materials with different dielectric constant [3]. Photonic crystals can improve the performance of optical devices such as waveguides, filters, light emitting diodes, and semiconductor lasers. Metallic periodic structures are also useful in improving radiation efficiency and patterns of antennas in microwave and millimeter wave ranges $[4,5]$. Thus periodic structures are expected to be useful in manipulating $\mathrm{THz}$ waves and could act as functional devices in a $\mathrm{THz}$ frequency range [6-10].

Usually, planar metallic structures on solid or flexible films have been fabricated by a photolithography technique including nine steps: making a photo-mask, cleaning the substrate, spin-coating the photo-resist, baking, exposure, development, post-baking, metal evaporation (etching) and resist removal. The processes demand a long task time and high cost.

Recently, printing techniques have been widely used in fabricating electronic components on polymer films. The printing method has only 3 steps: cleaning substrate, ink-jet printing, and heating. The last process lets silver nanoparticles be sintered together and thus a printed sample becomes metallic. The printing method reduces processing time and effort for fabrication of planar type metallic structures on films. Walter et al., reported $\mathrm{THz}$ transmission of periodic arrays of split ring resonators (SRRs) printed on a polyimide substrate with ink including silver nanoparticles [11]. Hangyo et al., also reported fabrication of wire-grid structures and SRRs on high-resistivity $\mathrm{Si}$ substrates by using a super-fine ink-jet printer with silver paste ink [12]. The printed structures act a good polarizer and filter. Thus the printing method can be useful in fabricating periodic structures to work in a $\mathrm{THz}$ range. It is interesting to print the periodic structures on conventional photo-papers to have no absorption in a $\mathrm{THz}$ range. A photo-paper is also flexible as well as cheap. Moreover, surface treatments such

\footnotetext{
*Corresponding author: cskee@gist.ac.kr

Color versions of one or more of the figures in this paper are available online.
} 
as plasma or ultrasonic cleaning are not required, unlike Si-substrates and polymide substrates.

In this paper, we report fabrication of a one-dimensional arrays of metallic wires and two-dimensional arrays of metallic SRRs by using an ink-jet printer and a conductive ink with silver nanoparticles. The structures were printed on a conventional photo-paper. The $\mathrm{THz}$ spectral responses are measured by a home-made THz-TDS system. The measured spectra show that the metallic wire array can act as a $\mathrm{THz}$ polarizer and the SSR array can act as a $\mathrm{THz}$ band-stop filter in a certain $\mathrm{THz}$ range.

\section{FABRICATION}

A high-dpi (dots-per-inch) resolution drop-on-demand printer and a conductive ink were employed to fabricate metallic periodic arrays on a conventional photo-paper. We

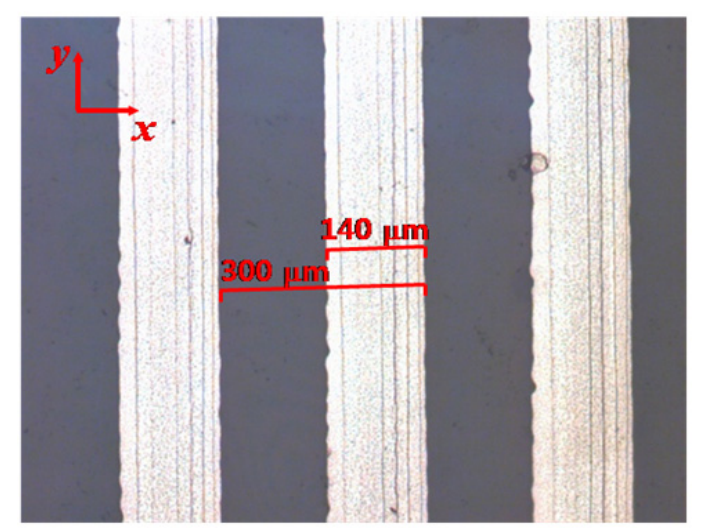

(a)

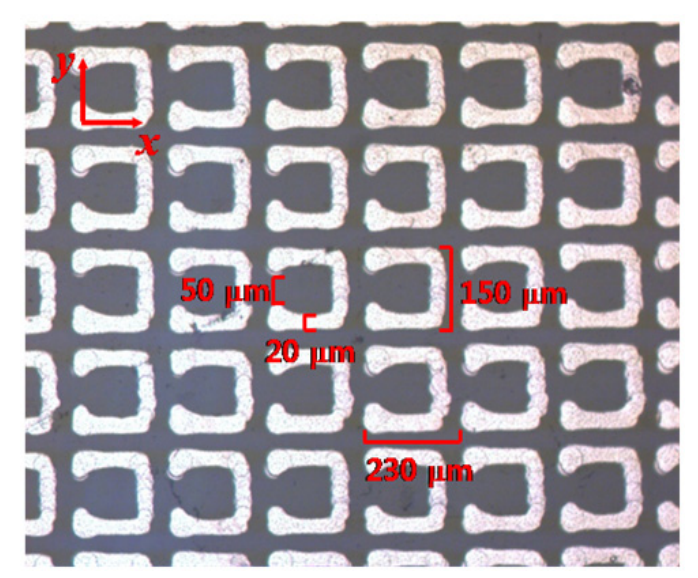

(b)

FIG. 1. Microscopic image of a printed one-dimensional array of wires with the period of $300 \mu \mathrm{m}$ and the width of $140 \mu \mathrm{m}$ (a) and a two-dimensional array of single split ring resonators (b). The one side length of a SSR is about $150 \mu \mathrm{m}$. The line width and the gap size are about $20 \mu \mathrm{m}$ and $50 \mu \mathrm{m}$, respectively. The period of the array is about $230 \mu \mathrm{m}$. printed a one-dimensional array of wires on a general-purpose photo paper with an ink containing silver nano-particles (DGP-40LT-15C) by using an ink-jet printer (DMP-2831). The samples were placed on a hot plate at $130^{\circ} \mathrm{C}$ during 30 minutes for the sintering of silver nano-particles.

The feature resolution of a printed structure depends on a nozzle to jet ink drops and compositions of ink. We have tried to find conditions to give a good feature resolution by varying both the weight percent of $\mathrm{Ag}$ in the ink and temperature and bias-voltage of a nozzle. After a lot of trial and error, we achieved $20 \mu \mathrm{m}$-feature resolution that is good for printing periodic arrays to work in a $\mathrm{THz}$ frequency range under the conditions that temperature and bias-voltage of a nozzle are about $30^{\circ} \mathrm{C}$ and $16 \mathrm{~V}$, respectively, and the weight percent of $\mathrm{Ag}$ is 32.0. However, printed arrays with $20 \mu \mathrm{m}$-feature resolution do not exhibit metallic properties because few Ag nano-particles are sintered in printed $20 \mu \mathrm{m}$-lines. To improve probability of sintering between Ag nano-particles, we iterated printing on the same place maintaining the $20 \mu \mathrm{m}$-feature resolution. We have found that four iteration printing is enough to make structures which exhibit metallic properties.

Fig. 1(a) shows the printed one-dimensional array of wires with a period of $300 \mu \mathrm{m}$ and width of $140 \mu \mathrm{m}$. A two-dimensional array of SRRs printed on the photo paper is shown in Fig. 1(b). The one side length of a SSR is about $150 \mu \mathrm{m}$. The line width and the gap size are about $20 \mu \mathrm{m}$ and $50 \mu \mathrm{m}$, respectively. The period of the array is about $230 \mu \mathrm{m}$. The printed sample sizes are about $1.0 \times$ $1.0 \mathrm{~cm}^{2}$.

\section{RESULTS AND DISCUSSION}

A home-made THz-TDS system was employed to measure the transmission properties of the planar metallic periodic structures. The schematic view of the experimental set up is represented in Fig. 2.[13] For the generation $\mathrm{THz}$ pulses, a $p$-type (100) InAs wafer with about $10^{16} / \mathrm{cm}^{3}$

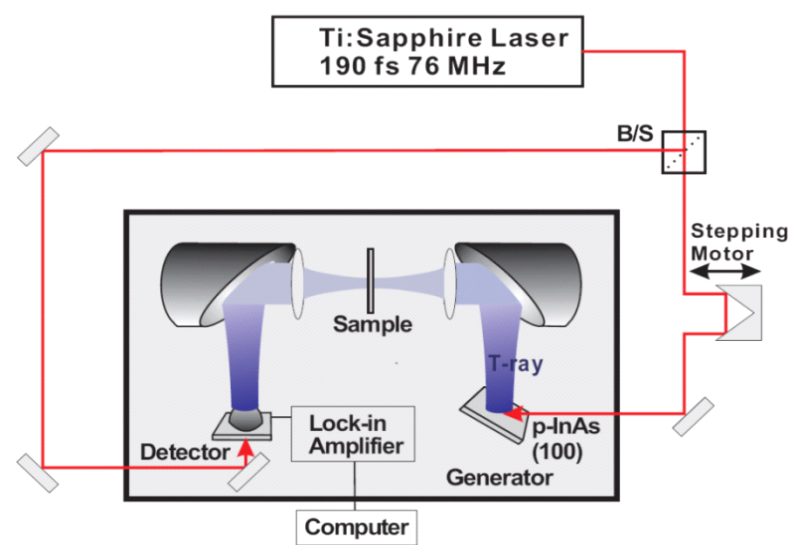

FIG. 2. Schematic view of a home-made terahertz-time domain spectroscopy system employed in experiments. 
carrier concentration and a femtosecond laser with 76 $\mathrm{MHz}$ repetition rate and a $190 \mathrm{fs}$ pulse width were used. The average power of the input laser is 1.3 watts and its beam diameter on the InAs wafer is $4 \mathrm{~mm}$. The $5-\mu \mathrm{m}$ gap dipole antenna on the low temperature grown GaAs wafer was used to detect $\mathrm{THz}$ pulses.

Fig. 3 shows the transmission spectra of the one-dimensional wire array for $\mathrm{THz}$ pulses with polarizations perpendicular ( $x$-polarized) and parallel ( $y$-polarized) to the wire when $\mathrm{THz}$ pulses are normally incident on the sample. The transmission spectrum of the paper was presented as a reference spectrum. The transmittances of the sample were normalized to that of the paper. One can see that the planar onedimensional wire array prevents the $y$-polarized $\mathrm{THz}$ waves with frequencies below $0.35 \mathrm{THz}$ from transmitting through the sample, while it does not affect the $x$-polarized $\mathrm{THz}$ waves. The $y$-polarized waves induce currents in the wires and thus the wire array structure acts effectively as a metal for the waves. However, for the $x$-polarized waves, the structure does not act as a metal but, in effect, as a lossy dielectric because currents are not induced by the $x$-polarized waves. In fact, due to the polarization dependent property, a one-dimensional metallic wire array has been used as a polarizer for waves with wavelengths much longer than the period of the array [14, 15].

To test the ability of our sample as a $\mathrm{THz}$ polarizer, we calculated the ratio of transmittances of $x$ - and $y$-polarized THz waves, $P_{x y}=T_{x} / T_{y}$, shown in Fig. 4. $P_{x y}$ calculated from transmittance data is depicted in Fig. 3. The dashed line indicates $P_{x y}=3 . T_{x}$ is larger than three times $T_{y}$ in a frequency range between 0.1 and $0.4 \mathrm{THz}$. Thus the printed wire array could be a polarizer in that range.

Fig. 5 shows the transmission spectra of a two-dimensional array of SRRs for the $x$ - and $y$-polarized THz waves. The dashed line presents the transmission spectrum of a paper used as a substrate. One can see that the SRR array exhibits

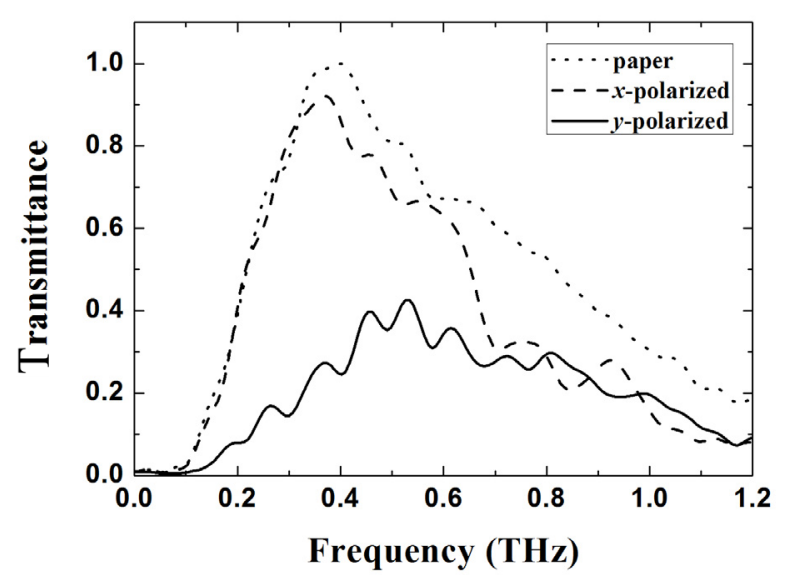

FIG. 3. Transmission spectra of the one-dimensional wire array for the $x$ - and the $y$-polarized $\mathrm{THz}$ waves when $\mathrm{THz}$ waves are normally incident on the sample. The transmission spectrum of the paper was presented as a reference spectrum. a transmission dip around $0.325 \mathrm{THz}$, for the $y$-polarized wave. It has been demonstrated that the $y$-polarized wave induces a current circulating along a SRR, and a magnetic property with negative permeability. Thus, a SRR array strongly reflects an electromagnetic wave in a frequency range where permeability is negative. Our printed sample has negative permeability around $0.325 \mathrm{THz}$ [16].

The SRR structure has been also modeled to be a $L C$ resonant circuit because the metallic line of a SRR gives rise to an inductance and the gap of a SRR gives rise to a capacitance [17]. A $L C$ resonant circuit exhibits high impedance at the resonant frequency and thus electromagnetic waves are highly reflected from the SRR array structure around the resonant frequency which corresponds to a frequency range with negative permeability. The resonant characteristic of a SRR could make the SRR array a $\mathrm{THz}$ band stop filter.

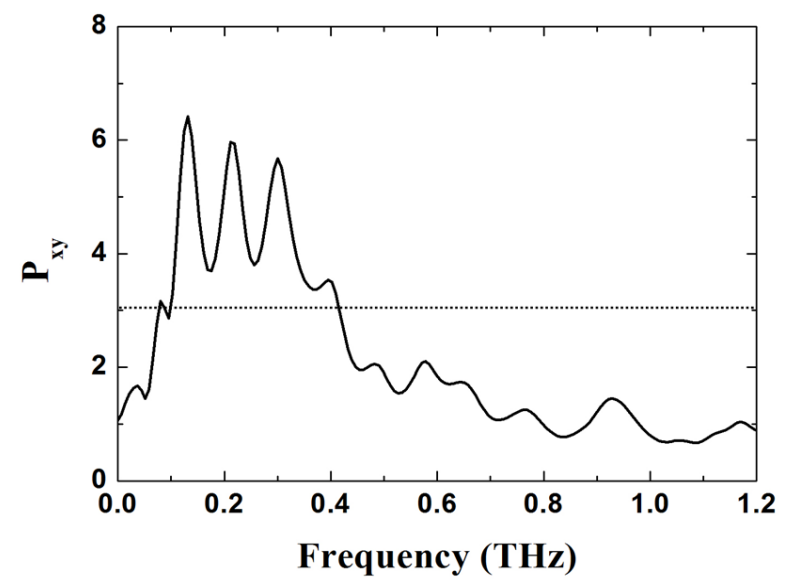

FIG. 4. The ratio of transmittances of $x$ - and $y$-polarized THz waves, $P_{x y}=T_{x} / T_{y}$, of the printed one-dimensional array of wires.

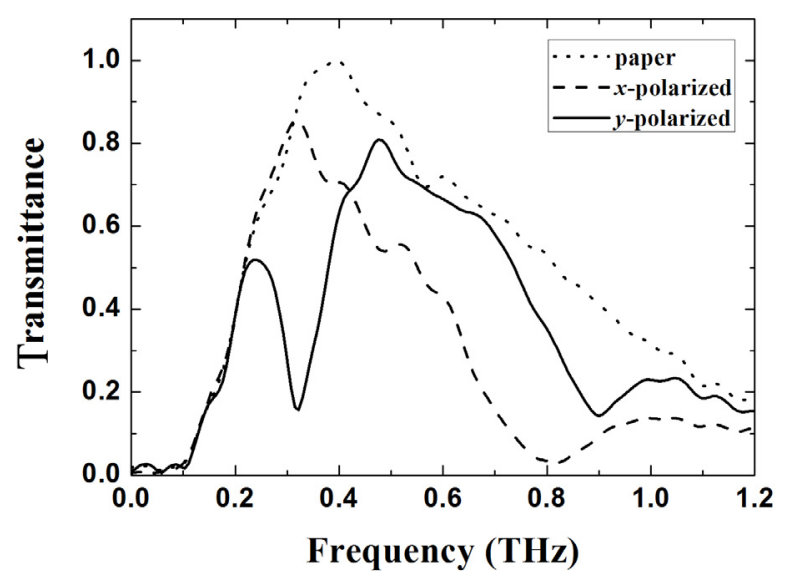

FIG. 5. Transmission spectra of a two-dimensional array of SRRs for the $x$ - and $y$-polarized THz waves. The dotted line presents the transmission spectrum of a substrate paper. 


\section{CONCLUSION}

A one-dimensional array of wires and a two-dimensional array of split ring resonators were printed on a photo-paper with an ink including silver nano-particles. The printed sample sizes are about $1.0 \times 1.0 \mathrm{~cm}^{2}$. The transmission measured by a THs system shows that the arrays of wires and split ring resonators could act as a polarizer and a band-stop filter in a THz region, respectively. The printing method could be a tool to realize various structures for functional $\mathrm{THz}$ devices.

\section{ACKNOWLEDGMENT}

This work was supported by the National Research Foundation Grant funded by the Korea Government (20100014291) and Photonics 2020 research project through a grant provided by GIST in 2010.

\section{REFERENCES}

1. S. Dexheimer, Terahertz Spectroscopy: Principles and Applications (Taylor \& Francis, London, UK, 2007).

2. Y. S. Lee, Principles of Terahertz Science and Technology (Springer, New York, USA, 2008).

3. J. Joannopoulos, S. Johnson, R. Meade, and J. Winn, Photonic Crystals: Molding the Flow of Light (Princeton University Press, Princeton, NJ, USA, 1995).

4. E. R. Brown, C. D. Parker, and E. Yablonovitch, "Radiation properties of a planar antenna on a photonic crystal substrate," J. Opt. Soc. Am. B 10, 404-407 (1993).

5. R. Gonzalo, P. de Maagt, and M. Sorolla, "Enhanced patch-antenna performance by suppressing surface waves using photonic-bandgap substrate," IEEE Trans. Microw. Theory Tech. 47, 2131-2138 (1999).
6. Y. Zhao and D. Grischkowsky, "Terahertz demonstrations of effectively two-dimensional photonic bandgap structures," Opt. Lett. 31, 1534-1536 (2006).

7. T. Prasad, V. L. Colvin, Z. Jian, and D. M. Mittleman, "Superprism effect in a metal-clad terahertz photonic crystal slab," Opt. Lett. 32, 683-685 (2007).

8. Y. Zhao and D. Grischkowsky, "2-D terahertz metallic photonic crystals in parallel-plate waveguides," IEEE Trans. Microw. Theory Tech. 55, 656-663 (2007)

9. H. Han, H. Park, M. Cho, and J. Kim, "Terahertz pulse propagation in a plastic photonic crystal fiber," Appl. Phys. Lett. 80, 2634-2636 (2002).

10. S. Kim, C.-S. Kee, and J. Lee, "Single-mode condition and dispersion of terahertz photonic crystal fiber," J. Opt. Soc. Korea 11, 97-100 (2007).

11. M. Walther, A. Ortner, H. Meier, U. Löffelmann, P. J. Smith, and J. G. Korvink, "Terahertz metamaterials fabricated by inkjet printing,” Appl. Phys. Lett. 95, 251107 (2009).

12. K. Takano, T. Kawabata, C. F. Hsieh, K. Akiyama, F. Miyamaru, Y. Abe, Y. Tokuda, R. P. Pan, C. L. Pan, and M. Hangyo, "Fabrication of terahertz planar metamaterials using a super-fine ink-jet printer," Appl. Phys. Exp. 3, 016701 (2010).

13. C. Kang, C.-S. Kee, I. B. Sohn, and J. Lee, "Spectral properties of THz-periodic metallic structures," J. Opt. Soc. Korea 12, 196-199 (2008).

14. A. E. Costley, K. H. Hursey, G. F. Neill, and J. M. Wald, "Free-standing fine-wire grids: their manufacture, performance, and use at millimeter and submillimeter wavelengths," J. Opt. Soc. Am. 67, 979-981 (1977).

15. C. L. Mok, W. G. Chambers, T. J. Parker, and A. E. Costley, "Far-infrared performance and application of freestanding grids wound from $5 \mu \mathrm{m}$ diameter tungsten wire," Infrared Phys. 19, 437-442 (1979).

16. T. J. Yen, W. J. Padilla, N. Fang, D. C. Vier, D. R. Smith, J. B. Pendry, D. N. Basov, and X. Zhang, "Terahertz magnetic response from artificial materials," Science $\mathbf{3 0 3}$, 1494-1496 (2004).

17. R. Marqués, F. Mesa, J. Martel, and F. Medina, "Comparative analysis of edge- and broadside- coupled split ring resonators for metamaterial design - theory and experiments," IEEE Transactions on Antennas and Propagation 51, 25722581 (2003). 